[20] S. Rothberg et al, ARS Journal, 28, 762 (1958).

[21] L. A. Barry, CEP (1958), Oct. 152.

[22] R. W. Warfield, ARS Journal, 30, 427. (1960).

[23] W. G. Strunk, CEP (1958), July, 45.

[24] В. И. Михеева в Е. М. Феднева, Изв. АН CCCP, OXH (1956), 902; H. I. Schlesinger et al, J. A. C. S., 75, 195 (1953); H. C. Brown et al, J. A. C. S., 80, 1552 (1958).

[25] R. Köster u. K. Ziegler, ang. Chem., 69, 94 (1957).

[26] 錢学森, ARS Journal, 23, 17 (1953).

[27] 岩間 樹, 山崎媇六, 工业化学杂志, 63，1879 (1960).

[28] R. C. Harshman, ARS Journal, 27, 398 (1958).

[28a] J. Buchanan et al, IEC, 48, 725 (1956).

[29] E. Mishuck \& L. T. Carleton, IEC, 52, 754
(1960).

[29a] A. M. Hoffman, IEC, 52, 781 (1950).

[30] C \& EN, Dec, 29, (1958) 26.

[31] C \& EN, Dec 7, (1959) 62.

[32] Chem. Week, Jan. 14, (1961), 34.

[33] A. B. Burg, ang. Chem., 72, 183 (1960).

[34] Chem. Eng., Sept. 5, 72 (1960).

[35] Aero/Space Eng., June, 1959, p. 37.

[36] W. 1. Murbach \& A. Adicoff, IEC, 52, 772 (1960).

[37] M. A. Alperin \& G. P. Sutton, Advanced Propulsion Systems, p. 211, Pergamon Press, London (1959).

[38] G. S. Sutherland, ARS Journal, 29, 698 (1959).

[39]“錢学森, ARS Journal, 26, 559 (1956).

[40] R. W. Bussard, ARS Journal, 28, 223 (1958).

\title{
放射性同位素在研究金屬中偏析和 非金屬夾雜物方面的应用
}

\section{李 代 鍾}

放射性同位素在治金研究工作中广泛应 用，还只是最近十余年来的事情。目前人造 的放射性同位素已經超过一千种，但是有好 多放射性同位素的半寒期很短，不适用于䂰 究工作。冶金中重要的元素如氧、氮、鋁、 敛等，就没有适当的放射性同位素。

放射性同位素在治金研究中最主要的用 途是作为示踪剂，因为示踪原子的方法具有 m灵敏度高、易于分辩等其他研究方法所不能

睲的特点。

\section{研究全中中㣂析的自射楼照相方法}

研究金属中偏析最有利的方法是利用放 淉性同位素自射綫炤相。由于放射性元素在
样品中分布的位置不同，因此它們的射綫和 箝胶片作用后，出現了相应的黑白影像。这 种乳胶片的特点是銀盐的浓度較高，粒子堹 細；郎所謂核子孚胶; 也有用 $\mathrm{X}$ 光底片来进 行自射綫照相的。

对金属进行自射綫照相,要求样品很溥, 而表面光洁度高。因为金属內部的射綫也能 穿透出来与乳胶发生作用, 从而使所得的图 案不能复实地反映表面的情况。特別是对放 射能量䡆高的同位素进行照相时，要求样品 漸到若干个微米。并要特別注意避堍由于样 品上的油迹、顏色、刻划等所造成的伪效应。

放射性同位素加入金属試样的方法有二 个：一是当金属熔炼时加入（或者在高温时 
渗入)。一是将需要研究的試样直接放在粒 子源中照射”，使被研究的元素激活成为放射 性同位素。在熔炼时加入放射性同位素的方 法是最常用的，加入的同位素的半衰期宜长 一些，其存在形式(元素状态或化合物状态) 也須考虑，否則同位菜的損失很大或者达不 到所要求的效果。試样直接在粒子源中照射 活化的方法, 特別适用于牛衰期短的同位素。 因为样品中元素对粒子的俘获截面不同，所 以选择适当的炤射时間，样品中被研究的元 素便能由同位素核反应 (即被照射物稹与生 成的放射性同位素属于同一元素) 或非同位 菜核反应（郎被照射物稹与生成的放射性同 位素不属于同一元素)而变成放射性同位素， 再按一般方法进行放射性照象或測量。如有 人研究嗍在鉄和鋼中的分布时，便是将样品 和乳胶底片一起放置于原子堆中, 由 ${ }_{5} \mathrm{~B}^{10}(n$, $\alpha)_{3} \mathrm{Li}^{7}$ 核反应的 $\alpha$ 射綫便在乳胶底片上形成 了影象。但这种方法只有少数的这种核反应 可以利用，所以限于很特殊的情况。

下面是一个在金属中加入气体问位素作 自射綫照相的例子。 Б. И. Брук 和 Г. И. Николаев 在研究容在鋯和鈦中的分布情况 时, 先使気水在真空中成为水蘶气, 抹使它 在鋯屑表面分解，生成的氛再使之渗入被湖 究的鋯、鈦样品中, 然后如常用方法作自射 綫照相，得到澄在这些金属中分布的很清晰 的图象(图 1 及图 2)。

自射綫照相方法不仅可以作一般的定性 鑑定，利且可以作定量測定。定量方法一般 有两个: 一是測定乳胶片上射綫印痕的数目 来确定样品中同位素的含量; 一是由測量照 相底片上图案的黑度来确定其元”素的含量。 后一方法也是測定金属中偏析的最常用的方

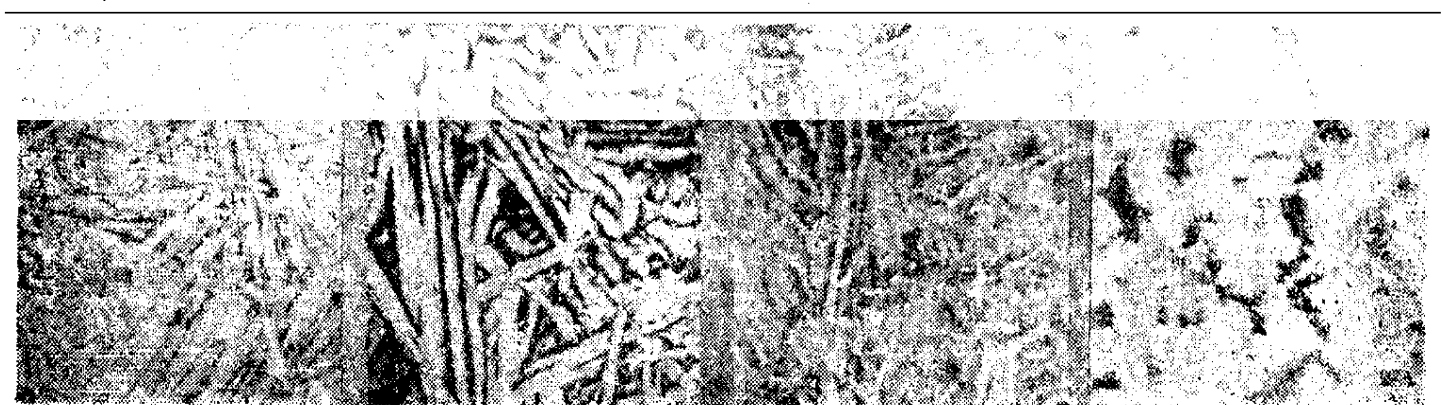

A B

图 1 工业純金属鈦在 $980^{\circ} \mathrm{C}$ 以氮飽和后, 在空气中冷却。
A

图 2 工业純金属鋯在 $100^{\circ} \mathrm{C}$ 以分能和后， 在空气中佮却。
A. 金相显微照相 $\times 100$
B. 自射䋐照相 $\times 100$
A. 金相显微照相 $\times 200$
B. 自射綫炤相 $\times 300$

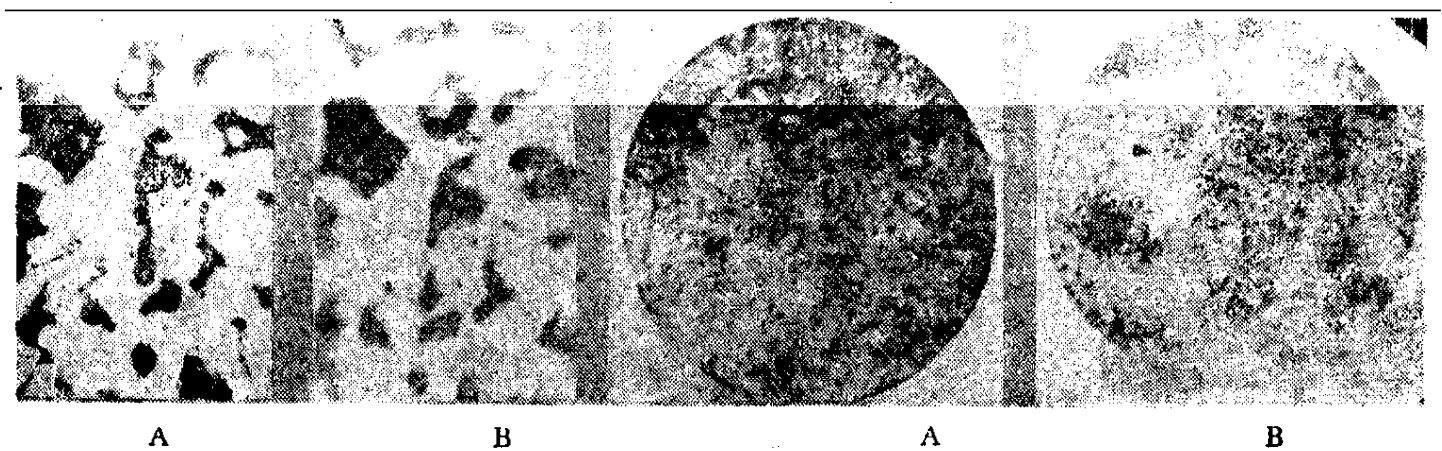

图 320 号鋼高頻淬火前的組織 A. 。金相显微照相 $\times 135$
B. 自射綫照相 $\times 134$
图 420 号鋼在 $1300^{\circ} \mathrm{C}$ 高頻淬火后組織 A. 金相显微照相 $\times 135$ B. 自射䋐照相 $\times 135$ 
法。

当然，自射綫照相方法目前还不是十分 完备的，它在技术上还有一些問題，这也正 是目前継嚗在研究改进的課題。另外，它的 分辦力不高, 一般在 $2-3$ 微米左右。

\section{用放射性同位䅅研究金属的偏析}

目前无諭在龬鉄或者有色金属和稀有金 属等的研究方面，都已广泛地应用了放射性 同位素自射綫照相的方法, 抹且得出了許多 重要的結果。

了解金属中元素的微覌偏析是政究金属 扩散性质及其他机理的重要环节。我們知 道，銅經高頻淬火后，碳在組織中的分布与 用其他淬火方法不同，这由 X射綫图案已經 証明。但是 X 射綫方法不能决定高頻淬火前 后組織中碳分布的浓度变化。自射綫照相的 特点可以从比較图 $3 \mathrm{~A} 、 \mathrm{~B}$ 和图 $4 \mathrm{~A} 、 \mathrm{~B}$ 的四 张照片中清楚地看出来。图 $3 \mathrm{~A} 、 \mathrm{~B}$ 是末淬火 前的組糡, 图 4 A、B 是 $1,300^{\circ} \mathrm{C}$ 高頻淬火后 的組糡。由自射綫照相中可以看出在淬火层 中碳稍微有些偏析，但是金相照片就显示不 出碳的这种不均匀变化。

合金中的枝晶偏析和比重偏析常常是影 响合金性能的重要因素，了解偏析的情况从 而来决定热处理制度，这对于生产是很有意 义的。又如用 $\mathrm{Ca}^{45}$ 研究銔在鎂合金中的偏 析，当銔在鎂中的含量为万分之几时是均匀 分布，到千分之几时，有明显的树枝状組 織，而釷是富集于树枝軸間，含䤮愈高，則 这种偏析情兄就永严重。这种偏析与合金冷 却速度、形变量、退火温度都有直接关系。 当快冷时，㺰不产生偏析，合金結构是均匀 的; 中等的冷却速度时，形成很不均匀的枝 晶偏析；最慢冷却速度时，釷的微观分布又
显然比較均与。另外，这种合金以 $50 \%$ 的 形变量佮不足以消除鑄造的树枝状結构，要 使树枝状結构全部消除，須要增高形变量至 75- $80 \%$ 。在热处理时，鑄造态的合金加热 至 $400^{\circ} \mathrm{C}$ ，保温 24 小时，㸗保持树枝状組 織，在 $500^{\circ} \mathrm{C}$ 退火料保温同样时間，則漸漸 消除树枝結构，鋢分布均一，但是加热至 $600^{\circ} \mathrm{C}$ 时，鋁又有些局部聚集。

金属中由于元素或化合物的不均匀分布 或局部集中对机械性能有很大影响，在这一 方面也用自射綫照相方法做过許多工作。例 如关于 $37 \mathrm{Cr}-\mathrm{Ni} 3$ 鋼的脆性断裂問題, 研 究結果指出：增加鋼门鉛量則增加脆性断裂 的倾向。鍽样过热后慢冷，則有粗大的硫化 物成連續网状分布于奥氏体晶界，过热后快 冷时，硫化物就呈很細小的夹杂物分布于整 个晶粒中。所以鋼加热后快冷不曾产生脆 性，而慢冷时硫化物沿奥氏体晶界析出，这 是使鋼产生脆性的基本原因。

用放射性同位素可以来考察某些元素在 金属中的分布或存在形式。如氢对鋯、鈦的 性能有值接的影响，因此 Б. И. Брук 等应

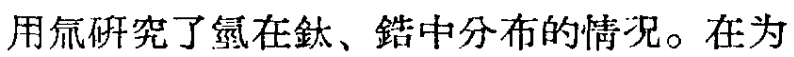
氛所能和的純鈦照片（图 1B）上表明，灰白 的針状組糡为含氮甚低的 $\boldsymbol{\alpha}$ 相，而暗黑部分 为含緛很高的 $\alpha$ 相和鈦的氫化物的共唱，这 是以往用金相力法所不能可靠地判断的。灭 由为気能和的純鋯照片中 (图 2B)，分有于 晶界的为 $\alpha$ 相和鋯的绿化物的共晶。同时由 比䡥图 $1 \mathrm{~A}$ 与 $\mathrm{B}$ 及图 $2 \mathrm{~A}$ 与 $\mathrm{B}$ 四张图片可以 看出，鈦的自射綫照相組糡与金相照相組織 相同，而鋯的这两种照相組織就不一样，所 以也說明了 $\mathrm{Ti}-\mathrm{H}$ 系和 $\mathrm{Zr}-\mathrm{H}$ 系不是相同的 結构。稀土金属已甚广泛地加入鋼或合金中 来提高其机械性能或腐蝕性能。了解稀土在 
銅中或合金中存在的形式，从而閶明其机理， 这是放射性同位素能帮助很好解决的。曾經 以放射性鈰和釹做过稀土在鉻镍不銹鋼中的 分布。証明它們稍有些集中于枝晶間，这种 偏析在热处理后显著減弱。一般情况下，鈰 和鉝不沿晶界分布，也不呈非金属夹杂物出 現；而是有規則地分布于金属基体中。

总之，利用放射性同位素已經做了很多 研究金属中偏析的工作，虽然自射綫照相方 法还不是十分完善的，然而在目前还是㸴究 偏析的最有利的方法。

\section{用放射性同位素研究金属中的 非金属夹杂物}

用放射性同位素研究金属中非金属夹杂 物的絕大多数工作是在鋼中的非金属夹杂物 方面。

非金属夹杂物对㴑珠鋼的盾量有很严重 的影响，应用放射性同位素破究源珠鋼中夹 杂物的来源及其排除問題是有重要意义的。 1954 年, А. М. Самарин 等首先发表了用 放射性同位素研究滚珠鋼中非金属夹杂物的 来源的工作。他們将 $\mathrm{Ca}^{45}$ 混入熔炼及浇注 用的各种耐火材料中。熔炼試驗在 10 公斤 威应炉內进行，又在三吨电炉的噡炼条件下 驗核了其中的一部分試驗。結果指出，炉渣 不会进入咳珠鋼中成为非金属夹杂物。碱性 高頻炉炉祄也只有当鋼液被強烈氧化时，才 有些进入鋼中成为一部分夹杂物。而外来夹 杂物的主要来源是浇注用的耐火材料, 特別 来自盛鋼桶的内祄。这里的試驗結果指出， 鋼中的夹杂物有 5-9\% 来自出鋼槽, 有 $18 \%$ 来自盛鋼桶內祄。継后，他們在 20 吨 的碱性电炉中进行了治炼同样鋼种的試驗, 背定了銅中外来夹杂物主要来自盛鎆桶的內
祄。指出这些夹杂物虽然占全部夹杂物的百 分数不大，但是顆粒甚大。要诚少这些夹杂 物需用高鉊砖作盛鋼桶内祄。以后，A.M.

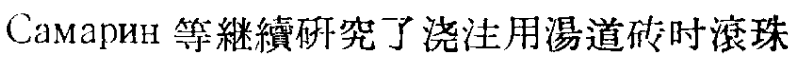
銅中非金属夹杂物的影响。所用的示踪剂仍 为 $\mathrm{Ca}^{45}$, 不过加入方法稍有改变。治炼和其 他試驗条件都与工厂生产情况相同，而湯道 磁分别用高岭士、高鋁士、石墨㕿士和普邀的 黏士做成。所得結果指出，鋼中外来夹杂物 含量没有超过总夹杂物的 $1 \%$ ，而且这个夹 杂物含量与所用浇注耐火材料的种类是没有 关系的，他們羭没有发現外来夹杂物在鋼鋁: 中分布的規律。有人在生产条件下研究过还 原渣和㴦珠鋼中夹杂物的关系，証实了从碱 性电炉出来的鋼液有被炉渣沾污的可能性。 在不同情况下，这种渣的夹杂物占总夹杂物 量的 0.001-10\%。当渣的化学成分相同时, 粘度高的渣对鋼的沾污影响較小。同时䚯为 鋼液在經过湯道时有一部分夹杂物被除去。

除了浐珠鋼外，对其他鋼种中夹杂物的 来源問題也进行过类似的㸴究。

綜合以往用放射性同位素对鋼中外来夹 杂物与炉渣夹杂物的一些㸴究, 可以归納为 下面几个要点: 1. 用放射性同位素作这方面 的研究，大都是工业挸模的試驗，放射性的 強度一次达儿百毫居里。虽然这些試驗的操 作比輘繁复, 但利㳊有什么特殊問題, 重要 的是.正确地选择試驗方法。2. 由于这些試驗 的条件比䡯复杂，因此他們的研究結果有些 是有局限性的，所以对于鋼中来自耐火材料 或炉渣的夹杂物的具体数字，没有啮遍的或 实际的意义，3. 有儿点結論是相京的, 郎:

（1）外来夾杂物在鋼錠中的分布没有什么規 律;（2）外来夹杂物占鋼: 隐夹杂物的含量: 不大, 但顆粒較大, 因此对氧化物的評級数 
有值接影响;（3）出鎡温度高时对耐火材料 的破坏也愈大。

鋼液出炉后的二次氧化对鋼中夹杂物的 影响及排除問題过去一直未得到很好的解 决。这也用放射性同位素作了研究，有人在 鎆液出炉之前不久加入 $\mathrm{Zr}^{85}$ 或 $\mathrm{Ta}^{182}$ ，然后 在出炉前和出炉后分別取銅样和椬样进行研 究。結果得出，在炉中被氧化的鋯在出鋼时 已从鋼液中除去; 在鋼液浇泩和結晶时被氧 化的鋯便大部分殘留于鋼中。但是氧化了的 鲥則几乎可以完全除去。这也就說明了銅中 的夹杂物主要是由于与氧亲和力最強的元秦 在鋼液浇泩和結晶时氧化而造成的。

\section{䊅語与展望}

近年来，虽然由于利用微束 X 射綫諳进 行了元素在金属中的偏析或分布的研究，从 而使金相学有了更梁的发展。然而利用放射 性同位素来砰究这些問題仍是十分必要的, 它可以帮助我們进入金相学的新天地中去。

在下列这些問題上都有必要利用放射性: 闰位素的方法来研究或作进一步探討。

1. 稀土元素目前在合金与合金鋼方面获 得了新的应用，然而稀土在合金中的許多机 理問題，佾未得到应有的訩識，有待研究解 决。

2. 其他微量元素也已广泛地被用来改进 铜或合金的各种性能，但某些杂貭元素在金 属材料中却起有害的影响，需要考察它們在: 金属中的分布及存在形式等等。
3. 研究金属在变形处理或热处理以后的 一些組織变化，例如新相的生成、溶解以及 其他变化。因为这些組糡变化往往是决定金 属材料性能好坏的关鍵。

4. 应用放射性同位素研究鎙中非金属夹 杂物的来源問題，虽然已做了許多工作，然 而結論很不一致。鋼出炉后的二次氧化問題 也了解得仍不透彻。这些都有必要作进一步 探討。

5. 淮确測定銅中非金属夹杂物的含量是 研究改进鋼稹㱖的一个方面，过去曾經进行 了許多工作，但所得結果我不令人滿意。对 于以往所用的測定方法的准确性也有异議。 所以对于准确测定鐝中央杂物含量的方法仍 是目前須要継縜研究的課題。可以利用放射 性同位素的示蹤作用来作些試探。

此外，放射性同位素还可用来决定某些 夹杂物的組成，考察某些元素或夹杂物在金 属中的偏析或分布、某些新相的組成、不闰 的冾却速度对金属結晶的影响等等。

当然，砤究金属中偏析和非金属夹杂物 还只是治金学中的一部分，另外象治金中的 扩散、治金物理化学、治金过程的控制、治 金分析等方面的研究，也都相当广泛地应用 了放射性同位素。

我国的治金工业正在飞速发展，实际生 产对治金科学提出了很多問題，因此应用放 射性同位素来帮助解决这些疑难，进一步推 动治金科学的发展，是目前迫切的任务。 